\title{
Implementação da comunicação médico-doente via endereço electrónico na consulta de MGF
}

Rita Pinhão,* Marlene Calisto,* Marta Arnaut Pimentel,* Rute Fernandes*

\section{RESUMO}

Introdução: A correspondência electrónica tem adquirido um espaço importante na troca de informação e nas relações interpessoais. A sua relevância e utilidade na área da saúde levaram à criação de orientações pelo Comité Permanente dos Médicos Europeus e pela American Medical Informatics Association para a correspondência electrónica nos cuidados ao utente.

Face às potencialidades da comunicação electrónica e ao facto de se desconhecer, em Portugal, a dimensão da sua utilização, considerou-se pertinente analisar a experiência de um médico de família (MF) que introduziu esta via de comunicação na sua prática clínica.

Objectivo: Com este trabalho pretende-se caracterizar os utentes utilizadores de endereço electrónico (e-mail) da lista de um MF, quanto ao sexo e idade, e conhecer os motivos pelos quais o utilizaram.

Tipo de Estudo: Estudo observacional, descritivo, transversal.

Local: Centro de Saúde de Cascais, USF Marginal.

População: Utentes que forneceram o e-mail de 15 de Dezembro de 2007 até 26 de Junho de 2008.

Métodos: Consulta de processos clínicos e dos e-mails recebidos. Base de dados e tratamento estatístico realizados no programa Microsoft Office Excel 2003.

Resultados: Da lista de utentes do MF, 283 (15,2\%) forneceram o e-mail, sendo $64,0 \%$ do sexo feminino. A média de idades foi $44,8( \pm 18,5)$ anos. Dos 283 utentes que forneceram o e-mail, 30,0\% usaram este meio de comunicação, o que corresponde a $5,0 \%$ da lista de utentes. Foram recebidas 204 mensagens. Os motivos mais frequentes foram: questões administrativas (36,9\%), informação sobre resultados de exames complementares de diagnóstico $(13,1 \%)$, dúvidas relacionadas com a saúde (11,2\%) e acompanhamento clínico (10,7\%).

Conclusões: Relativamente ao motivo de contacto, os resultados vão de encontro aos obtidos noutros estudos e são concordantes com as recomendações europeias e norte-americanas.

O e-mail poderá ser um importante complemento da consulta presencial, permitindo melhorar a qualidade dos cuidados prestados e a gestão do tempo dos profissionais de saúde.

Palavras-chave: Correio Electrónico; Relação Médico-Doente; Comunicação; Cuidados de Saúde Primários.

\section{INTRODUÇÃO}

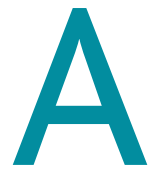
comunicação médico-doente é a base para o estabelecimento de uma boa relação clínica. Para além da consulta presencial, existem outras formas de comunicação na área da saúde, tais como por carta e por telefone.

Nos últimos anos, a Internet, através da correspon-

* Médica interna do $2^{\circ}$ Ano de Medicina Geral e Familiar Centro de Saúde Cascais, USF Marginal dência electrónica, adquiriu um espaço importante na troca de informação e nas relações interpessoais. Nos Estados Unidos da América (EUA), em 1993 estimavase que cerca de 12 a 20 milhões de pessoas tinham acesso ao $e$-mail e em 1999 já este número tinha excedido os 100 milhões. ${ }^{1}$ Em Portugal, o acesso à Internet a partir do lar aumentou mais de 18 vezes nos últimos dez anos, passando de 2,4\% em 1997 para 44,6\% em 2007. ${ }^{2}$

O uso do correio electrónico, na prática clínica, é um 
fenómeno recente. A palavra-chave «Electronic Mail» foi apenas introduzida em 2003 na base de dados da $\mathrm{Me}$ dical Subject Headings (MeSH). ${ }^{3}$

Têm surgido vários trabalhos publicados para estudar esta forma de comunicação nos cuidados de saúde primários (CSP). No estudo de Moyer C. et al, realizado em dois grandes centros de CSP nos EUA, com 476 doentes, concluiu-se que mais de metade dos doentes usava regularmente o correio electrónico. Destes, 10,5\% já tinham comunicado com o seu médico por este meio e dois terços manifestaram interesse em fazê-lo. ${ }^{1} \mathrm{Ou}-$ tro estudo, de DeLuca e Enmark, verificou que 50\% dos doentes demonstraram interesse na comunicação com o médico por via electrónica e um terço considerou mudar de médico se lhe fosse disponibilizada esta forma de comunicação. ${ }^{4}$ Wearn A. et al., na Nova Zelândia, inquiriram médicos sobre este tema: em $80 \mathrm{MF}$, apenas $4 \%$ usava regularmente o correio electrónico com os seus doentes. A maioria demonstrou um grande interesse nesta forma de comunicação e considerou que seria vantajoso para a prática clínica, desde que suportado por guidelines ${ }^{3}$

A relevância deste tema levou à criação de orientações pelo Comité Permanente dos Médicos Europeus ${ }^{5}$ e pela American Medical Informatics Association ${ }^{6}$ para a correspondência electrónica nos cuidados ao utente, de forma a assegurar a qualidade da comunicação apoiada numa base ética sólida. As recomendações ${ }^{3,5-11}$ para a implementação da comunicação médico-doente por $e$-mail encontram-se resumidas no Quadro I.

Apesar das vantagens para médicos e utentes, a comunicação virtual em contexto clínico tem-se desenvolvido bastante aquém do esperado.

Face às potencialidades da comunicação electrónica e ao facto de se desconhecer, em Portugal, a sua utilização nos CSP, considerou-se pertinente analisar a experiência de um MF que introduziu esta via de comunicação na sua prática clínica, passando a propor aos seus utentes, na consulta, a comunicação por $e$-mail. Os que forneceram o seu endereço receberam um $e$-mail com as regras desta forma de comunicação (baseadas nas orientações europeias).

Com este trabalho pretende-se caracterizar, quanto ao sexo e idade, os utentes utilizadores de $e$-mailna lista de um MF e conhecer os motivos pelos quais utilizaram este meio de comunicação.

\section{MÉTODOS}

Realizou-se um estudo observacional, descritivo, transversal, através da consulta de processos clínicos existentes no programa Medicine One e os e-mails recebidos através do programa Outlook Express 6, de um MF a trabalhar numa Unidade de Saúde Familiar.

A base de dados e o tratamento estatístico foram realizados no programa Microsoft Office Excel 2003.

População: utentes que forneceram o $e$-mail, ao MF em análise, de 15 de Dezembro de 2007 até 26 de Junho de 2008 no Centro de Saúde de Cascais - USF Marginal.

Variáveis: idade (anos), sexo (feminino, masculino), motivos de comunicação (questões administrativas, informação sobre resultados de tratamentos, seguimento de medicação, dúvidas relacionadas com a saúde, acompanhamento clínico, informação sobre resultados de exames complementares de diagnóstico (ECD), retorno de informação de outras especialidades, doença aguda, motivos de cariz sensível, críticas ao MF e à USF, outros assuntos).

\section{RESULTADOS}

Dos 1.864 utentes da lista do MF, $15,2 \%$ ( $n=283$ ) forneceram o seu $e$-mail, no período em que decorreu o estudo. A média das idades deste grupo foi de 44,8 anos $( \pm 18,5)$. A idade mínima foi de oito anos e a máxima de 95 anos, $64 \%$ eram do sexo feminino.

Dos utentes que forneceram o $e$-mail, $30 \%(n=85)$ já comunicaram com o MF; destes $62 \%$ eram do sexo feminino. Das mulheres que forneceram o e-mail $29 \%$ $(n=53)$ comunicaram com MF e dos homens $31 \%$ $(n=32)$. Foram recebidas 204 mensagens.

Os 85 utilizadores do $e$-mail correspondem a $5 \%$ da lista total de utentes.

A média de idades dos utentes que comunicaram foi de 48,5 ( \pm 14,2 anos).

Os motivos mais frequentes foram: questões administrativas $(36,9 \%)$, informação sobre resultados de ECD (13,1\%), dúvidas relacionadas com saúde (11,2\%) e acompanhamento clínico (10,7\%). Os principais motivos de comunicação foram os mesmos para ambos os sexos (Quadro II).

\section{DISCUSSÃO}

Neste trabalho verificou-se que $5 \%$ dos utentes da lista do MF já comunicaram por $e$-mail. Num estudo rea- 


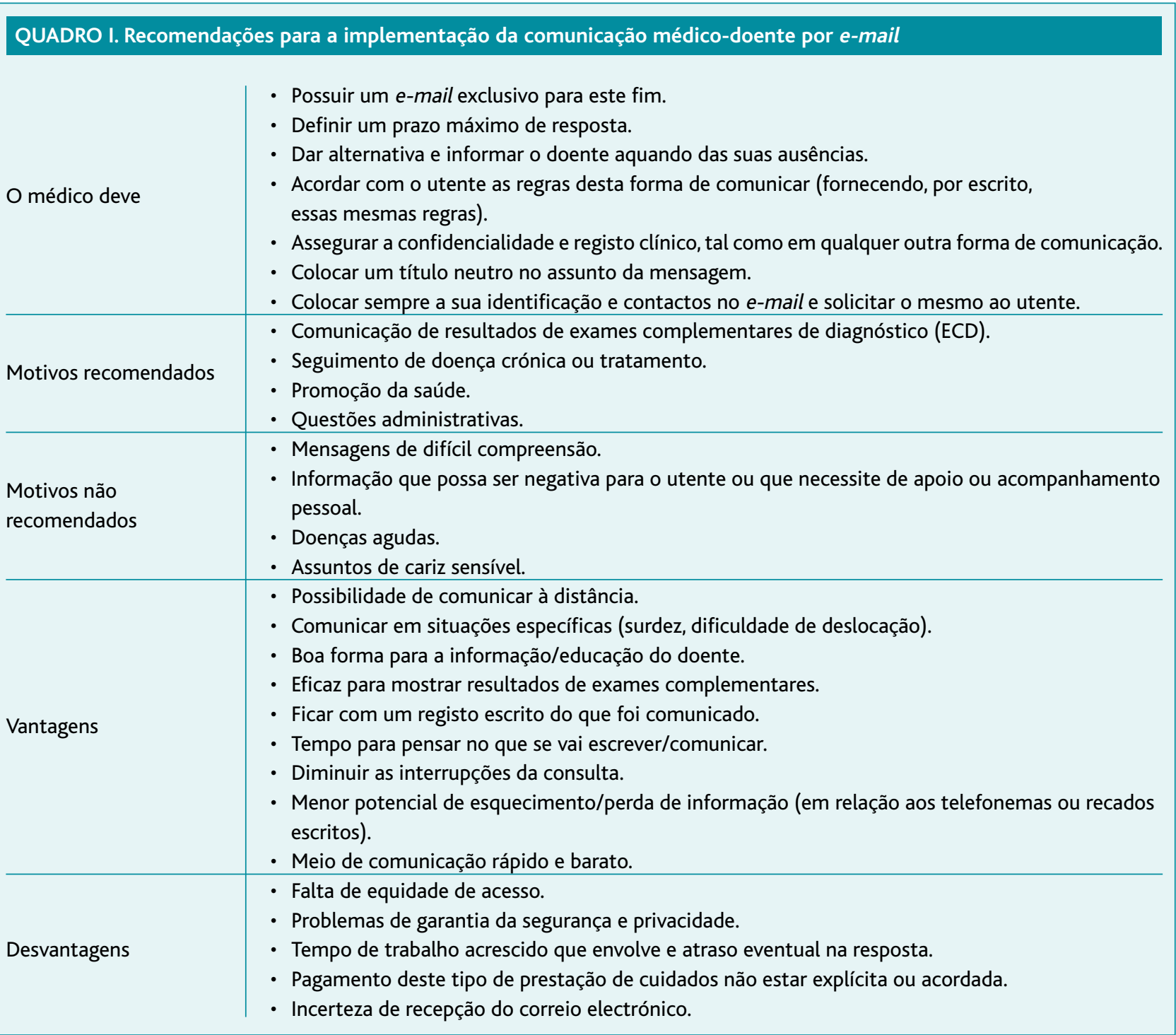

lizado em Boston, os MF também só comunicavam via $e$-mail com $5 \%$ dos utentes das suas listas. ${ }^{11}$

Na população estudada, os utentes eram maioritariamente do sexo feminino (64\%). Este resultado poder-se-á dever à existência de maior percentagem de mulheres na lista do MF e, também, a um maior consumo de consultas pelas utentes do sexo feminino.

Os motivos mais frequentes (questões administrativas, resultados de ECD, dúvidas relacionadas com saúde e acompanhamento clínico), são semelhantes aos encontrados noutros estudos ${ }^{7,11}$ e concordantes com as recomendações europeias e norte-americanas, para este tipo de comunicação. No entanto, verificou-se que $3,9 \%$ das mensagens recebidas reportavam-se a casos de doença aguda e $4,4 \%$ a motivos de cariz sensível, reflectindo haver ainda um uso inadequado do $e$-mail apesar das regras estabelecidas.

Os utentes do sexo masculino usaram mais o $e$-mail por motivo de doença aguda que o feminino, o que poderá advir de um menor uso dos serviços de saúde, sobretudo na forma presencial.

Como limitações deste estudo, podem-se apontar: 
QUADRO III. : Motivos de comunicação por e-mail $(n=206)$

\begin{tabular}{|c|c|c|c|c|c|c|}
\hline \multirow[b]{2}{*}{ Motivo } & \multicolumn{2}{|c|}{ Total } & \multicolumn{2}{|c|}{ Sexo Feminino } & \multicolumn{2}{|c|}{ Sexo masculino } \\
\hline & $\mathbf{n}$ & $\%$ & $\mathbf{n}$ & $\%$ & $\mathbf{n}$ & $\%$ \\
\hline Questões administrativas & 76 & 36,9 & 49 & 37,4 & 27 & 36 \\
\hline Informação sobre resultados ECD & 27 & 13,1 & 17 & 13 & 10 & 13,3 \\
\hline Outros assuntos & 23 & 11,2 & 13 & 9,9 & 10 & 13,3 \\
\hline Dúvidas relacionadas com saúde & 23 & 11,2 & 16 & 12,2 & 7 & 9,3 \\
\hline Acompanhamento clínico & 22 & 10,7 & 11 & 8,4 & 11 & 14,7 \\
\hline Motivos de cariz sensível & 9 & 4,4 & 9 & 6,9 & 0 & 0 \\
\hline Retorno de informação de outras especialidades & 9 & 4,4 & 6 & 4,6 & 3 & 4 \\
\hline Doença aguda & 8 & 3,9 & 3 & 2,3 & 5 & 6,7 \\
\hline Seguimento de medicação & 8 & 3,9 & 6 & 4,6 & 2 & 2,7 \\
\hline Críticas ao MF/USF & 1 & 0,49 & 1 & 0,8 & 0 & 0 \\
\hline Informação sobre resultados de tratamentos & 0 & 0 & 0 & 0 & 0 & 0 \\
\hline
\end{tabular}

incidir sobre a lista de apenas um MF; o curto período de tempo que decorreu desde a implementação do $e$-mail na consulta até à data do estudo (6 meses) e de terem sido avaliadas um pequeno número de características dos utentes e não se poder excluir um viés de classificação dos motivos dos e-mails. É, portanto, um estudo exploratório e, como tal, pode trazer algumas pistas para estudos futuros, nomeadamente: caracterizar os utentes quanto ao nível sócio-económico e de escolaridade; estudar se a implementação deste tipo de comunicação diminui as interrupções na consulta e os contactos directos e se poupa tempo ao binómio médico/administrativo e ao utente. Outra área de estudo possível é a avaliação da satisfação dos utentes a quem foi disponibilizada esta forma de comunicação.

Perante os resultados obtidos seria pertinente a criação de um e-mail administrativo para dar resposta às questões desta área, que foram as mais frequentes.

Apesar das regras estabelecidas inicialmente, ainda é necessário educar os utentes para evitar o uso inadequado do $e$-mail.

Esta nova forma de comunicação não se destina a substituir a consulta presencial, mas poderá ser um importante complemento desta, melhorando a qualidade dos cuidados prestados, em particular o acesso à equipa de saúde, e a gestão do tempo dos profissionais de saúde.

\section{REFERÊNCIAS BIBLIOGRÁFICAS}

1. Moyer CA, Stern DT, Dobias KS, Cox DT, Katz SJ. Bridging the electronic divide: patient and provider perspectives on e-mail communication in primary care. Am J Manag Care 2002 May; 8 (5): 427-33.

2. 10 Anos de Internet em Portugal: os números e os factos [página na internet]. Marktest. Disponível em: http://www.marktest.pt/marktest/default.asp?c=1210\&n=1897 [acedido em 2008/10/04].

3. Goodyear-Smith F, Wearn A, Everts $H$, Huggard P, Halliwell J. Pandora's electronic box: GP's reflect upon email communication with their patients. Inform Prim Care 2005; 13 (3): 195-202.

4. DeLuca JM, Enmark R. E-health: the changing model of healthcare. Front Health Serv Manage 2000 Fall; 17 (1): 3-15.

5. Ordem dos Médicos Portugueses. Orientações do Comité Permanente dos Médicos Europeus para a Correspondência Electrónica nos Cuidados ao Paciente.

6. Kane B, Sands DZ; The AMIA Internet Working Group, Task Force on Guidelines for the Use of Clnic-Patient Electronic Mail. Guidelines for the clinical use of electronic mail with patients. J Am Med Inform Assoc 1998 Jan-Feb; 5 (1): 104-11.

7. Leong SL, Gingrich D, Lewis PR, Mauger DT, George JH. Enhancing doctor-patient communication using e-mail: a pilot study. J Am Board Fam Pract 2005 May-Jun; 18 (3): 180-8.

8. Kassirer J. Patients, physicians and the internet. Health Aff (Milwood) 2000 Nov-Dec; 19 (6): 115-23.

9. Harrison JP, Lee A. The role of e-health in the changing health care environment. Nurs Econ 2006 Nov-Dec; 24 (6): 283-8.

10. Eads M. Virtual office visits: a reachable and reimbursable innovation. Fam Pract Manag 2007 Oct; 14 (9): 20-2.

11. Houston TK, Sands DZ, Jenckes MW, Ford DE. Experiences of patients who were early adopters of electronic communication with their physician: satisfaction, benefits, and concerns. Am J Manag Care 2004 Sep; 10 (9): 601-8.

As autoras declararam não possuir conflitos de interesses 
ENDEREÇO PARA CORRESPONDÊNCIA

Rita Pinhão

Unidade de Saúde Familiar Marginal

Rua Egas Moniz, Edf. Centro de Saúde de S.João do Estoril, piso 2

S. João do Estoril, 2765-458 Cascais

E-Mail: rita.pinhao@gmail.com
Recebido em 27/04/2009

Aceite para publicação em 08/12/2009

\section{ABSTRACT}

\section{DOCTOR-PATIENT COMMUNICATION THROUGH ELECTRONIC MAIL IN FAMILY PRACTICE CONSULTATION}

Introduction: E-mail correspondence has acquired its own place as a vital medium for information exchange and for people's relationships. Its applicability in health services has led to the creation of guidelines by the Permanent Committee of European Physicians and by the American Medical Informatics Association.

Bearing in mind the potential of e-mail and the fact that, in Portugal, the extent of its current use is unknown, we found it appropriate to study the experience of this sort of interaction on the practice of a Family Physician.

Aim: With this essay we intend to describe patients that use e-mail regarding their age and gender, and the purposes behind such use.

Type of study: Observational, descriptive, transversal.

Place: Cascais Community Health Center, USF Marginal.

Population: Patients that provided their e-mails, from December 15, 2007 to June 26, 2008.

Methodology: Analysis of medical records and e-mails received. Data base and statistical analysis was done using Microsoft Office Excel 2003.

Results: From the Physician's patient list, 283 (15.2\%) provided their e-mail addresses, $64.0 \%$ of whom were female. The age average was 44.8 ( \pm 18.5 ) years old.

Of the 283 patients that provided their e-mail addresses, $30 \%$ came to contact the Physician through its use, $5.0 \%$ of the Physician's total patients.

Two hundred and four e-mail messages were received. The most frequent subjects observed were: administrative questions $(36.9 \%)$, information regarding test results (13.1\%), questions on health related issues (11.2\%) and clinical follow up (10.7\%). Discussion: When we analyze the reasons for contact, results are similar to those obtained in other studies and are in conformity with European and North-American guidelines on this type of communication.

This new form of interaction may represent an important supplement to face to face consultation, improving the quality of care provided and health professionals' time management.

Keywords: Electronic Mail; Physician-patient Relations; Communication; Primary Health Care. 